\title{
Disseminated Echinococcosis of the Lung and Central Compartment of the Neck
}

\author{
Nischal Rajendra Pandya, MJ Paul, Lalit Choudhry, Birla Roy Gnanamuthu, Kochu Krishnan \\ Joshua Franklyn, Jyoti Prasad Kalita
}

\begin{abstract}
Echinococcosis is a ubiquitous zoonosis. The causal organism is a flat tapeworm whose larval form causes infestations in human beings, who are its accidental hosts. It is known to occur in all parts of the body with the highest incidence in the liver, followed by the lung. Hydatid cysts in the neck are rare by themselves. We report a case of hydatidosis of the lung and central compartment of the neck, in a man who presented with pulmonary symptoms only. Computerized tomogram of the chest had revealed a hydatid cyst in the left lung and another incidental hydatid cyst in the neck. He underwent successful excision of both the cysts in one sitting.
\end{abstract}

Keywords: Echinococcosis, Hydatid cyst, Neck, Lung.

How to cite this article: Pandya NR, Paul MJ, Choudhry L, Gnanamuthu BR, Krishnan K, Franklyn J, Kalita JP. Disseminated Echinococcosis of the Lung and Central Compartment of the Neck. World J Endoc Surg 2013;5(1): 25-27.

\section{Source of support: Nil}

Conflict of interest: None

\section{INTRODUCTION}

Hydatidosis is endemic in many parts of the world. It is transmitted from infected canines to ruminants and man. Man is an accidental intermediate host in the life cycle of this worm.

Hydatidosis is known to occur in all parts of the body with the highest incidence in the liver and lung. Hydatid cysts in the neck are rare. We report a case of hydatidosis in the central compartment of the neck adjacent to the thyroid gland, in a patient who presented with symptoms pertaining to pulmonary hydatidosis. We also discuss the differential diagnosis and evaluation of the neck lesion here.

\section{CASE REPORT}

A 46-year-old man presented with history of recurrent episodes of fever, cough, hemoptysis and left-sided chest pain of 4 months duration. Examination of the respiratory system was normal. However, there was an ill-defined fullness in the left side of his lower neck deep to the sternal head of the sternocleidomastoid muscle, detected incidentally on examination.

His chest X-ray showed a soft tissue opacity in the left upper and mid zones. Computerized tomogram (CT) of the chest revealed a $68 \times 58 \mathrm{~mm}$ hydatid cyst in the upper lobe of left lung (Fig. 1A) and an identical lesion of $42 \times 30 \mathrm{~mm}$ size in the left paratracheal region of the central compartment of the neck (Fig. 1B).

Focused ultrasound examination of the cysts in the neck and chest revealed trilaminar membranes in the cysts, diagnostic of a hydatid. The thyroid gland was normal. Preoperative fine needle aspiration examination of the cyst was not done because of the risk of disease dissemination and anaphylaxis. Serological tests were not considered necessary. CT showed no evidence of liver hydatid.

In view of the rarity of nonparenchymal neck hydatid, a differential diagnosis was considered. Cystic neck swellings include lesions that are congenital, inflammatory or neoplastic. Congenital lesions include branchial cleft cysts, thyroglossal duct cysts, ectopic thymus cysts, dermoid cysts, teratoid cysts, hemangiomas and lymphangiomas. Inflammatory lesions include abscesses, lymphadenitis and sialadenitis. Neoplastic lesions include cystic schwannomas, thyroid cysts or vascular angiomas.

Since, the lung hydatid required surgery and the neck lesion was nonparenchymal, PAIR (percutaneous aspiration, injection and reaspiration) was not indicated.

The patient was initiated on oral albendazole therapy for a week and then taken up for surgery. Through a posterolateral thoracotomy, the pulmonary hydatid was excised and capitonnage of the cavity was done. After this, the patient was repositioned and the cervical cyst exposed by a transverse cervical incision. The cyst in the central
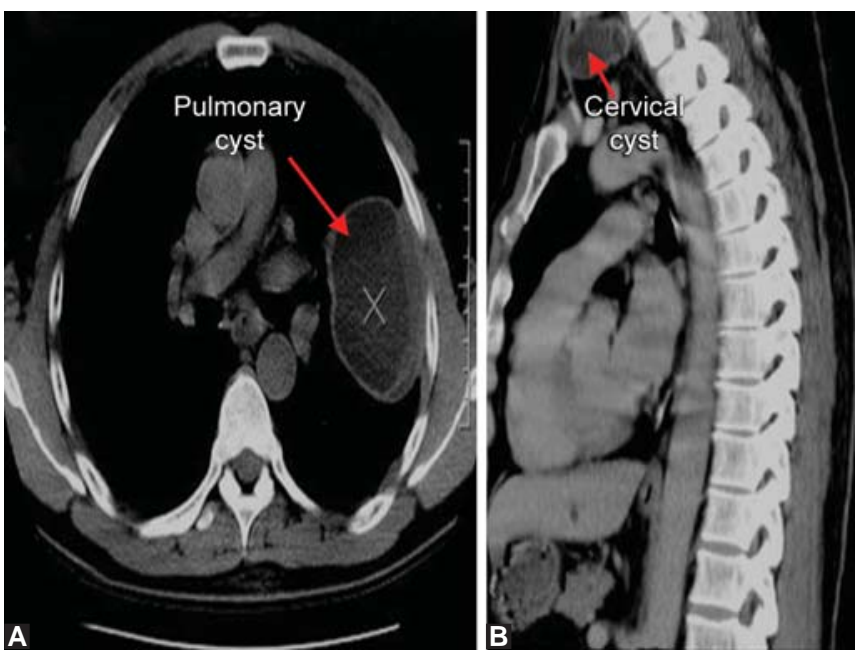

Figs 1A and B: Computerized tomography images showing the pulmonary and cervical hydatid cysts 
compartment of the neck, at the inferior pole of the left lobe of thyroid was excised with care to preserve the parathyroid glands, recurrent laryngeal nerve and the inferior thyroid vessels (Fig. 2). Spillage of the contents was avoided. The thyroid gland appeared to be otherwise normal and hence a thyroidectomy was not done. The histopathology report confirmed our diagnosis and the patient was treated with a 3-month regime of oral albendazole postoperatively. He had an uneventful postoperative recovery.

\section{DISCUSSION}

Hydatidosis is caused by the larva of the cestode echinococcus. Transmission to human beings occurs by the ingestion of eggs, while coming in contact with infected animals, their offal or while handling soil containing the eggs.

Carrion birds, coprophagic flies and arthropods are all known to act as mechanical vectors. It can be of cystic, alveolar or polycystic types. The eggs are partially resistant to dessication and can, therefore, remain viable for prolonged periods of time.

In humans, the liver is involved in $60 \%$ of the cases, lung in $30 \%$ and multiple organs in 20 to $30 \%$ of the cases. ${ }^{1,2}$ Primary involvement of extrahepatic sites without the involvement of the liver are attributed to the hydatid embryo gaining entry into the systemic circulation through the lacteals or by the direct inhalation of the embryophore. More often, the embryo is transmitted through the portal vein into the liver and from the liver to the other organs. Some of these embryos in the portal vein are even suspected to traverse the hepatic sinusoids into the systemic circulation without involving the liver. ${ }^{3}$

Simultaneously occurring primary hydatidosis of the neck and lung is a rare finding. Avcu et al state that hydatidosis of the head and neck are rare even in endemic areas. The reported sites in the head and neck region include the base of the skull, parapharyngeal space, supraclavicular region, the submandibular and the thyroid glands. ${ }^{4}$ The thyroid gland can be involved by the parasitic embryo either directly from the systemic circulation (primary type) or secondary to hepatic involvement (secondary type). The small diameter of the thyroid gland's vasculature prevents the incidence of seeding of the embryo into the gland. ${ }^{5}$

Mediastinal sites of primary hydatid include the superior mediastinum, pericardium and the heart. Mitra et al have reported a case of superior vena caval obstruction secondary to a mediastinal hydatid cyst presenting with a neck lump which was successfully excised. ${ }^{6}$ Cases of cervicothoracic hydatidosis have been described in the English literature albeit rarely. Such Pancoast hydatid cyst lesions are notorious in causing Horner's syndrome also. ${ }^{7}$
Though radiological investigations are often confirmatory, serological tests like hemagglutination, latex agglutination and echinococcus enzyme-linked immunosorbent assay test may help in arriving at a diagnosis.

Treatment by chemotherapy, surgical excision or aspiration and alcohol injection ${ }^{8}$ may be carried out depending on the characteristics of the cyst, although surgical excision is the treatment of choice for hydatidosis of the neck.

Complications, such as compression or infiltration of the surrounding tissue have been reported. The possibility of spontaneous rupture of the cyst leading to life threatening anaphylaxis should also be emphasized.

During surgery, injection of scolicidal agents into the cyst prior to opening it and avoidance of spillage of the contents could prevent dissemination and anaphylactic shock. Judicious use of steroids and ionotropes are to be considered in treating the anaphylaxis.

Cysts in the neck are excised along with the pericyst, whereas in the lungs, pericystectomy is reserved only for small peripherally located cysts (Figs 3A and B). In other commoner pulmonary locations, pericystectomy is best avoided since it leads to complications. Large cysts could warrant a lobectomy. Preoperative treatment with a benzimidazole drug which is continued for 3 months postoperatively is mandatory to prevent recurrence.

Primary level of prevention includes health education, sanitation, hygiene, animal vaccination and treatment of pets with scolicidals. Since, the hydatids are known to reoccur after surgery, routine postoperative surveillance is necessary.

\section{CONCLUSION}

Simultaneous primary hydatidosis in two distinct locations in this patient without the involvement of the liver is unique.

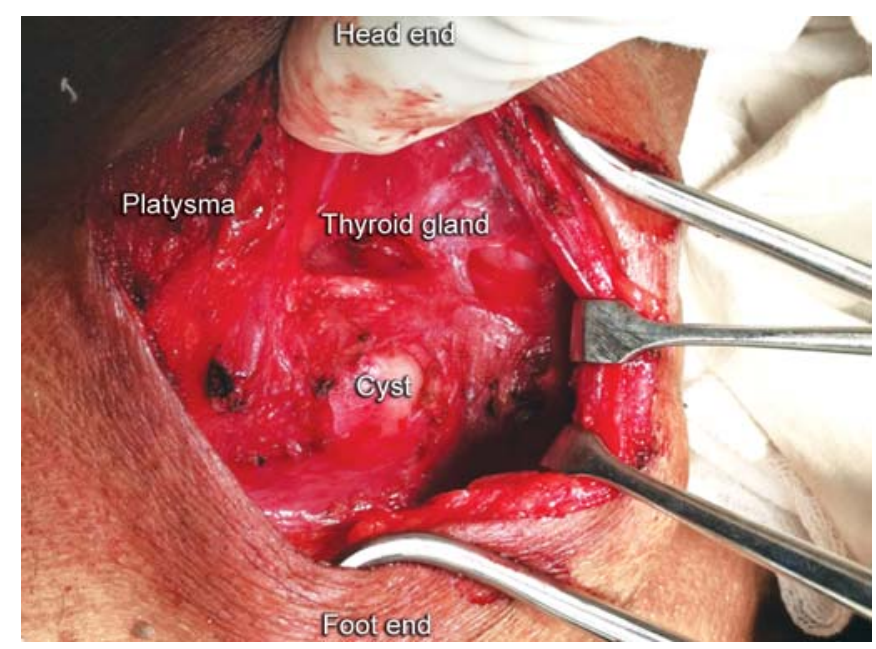

Fig. 2: Intraoperative photo of the cervical hydatid cyst 


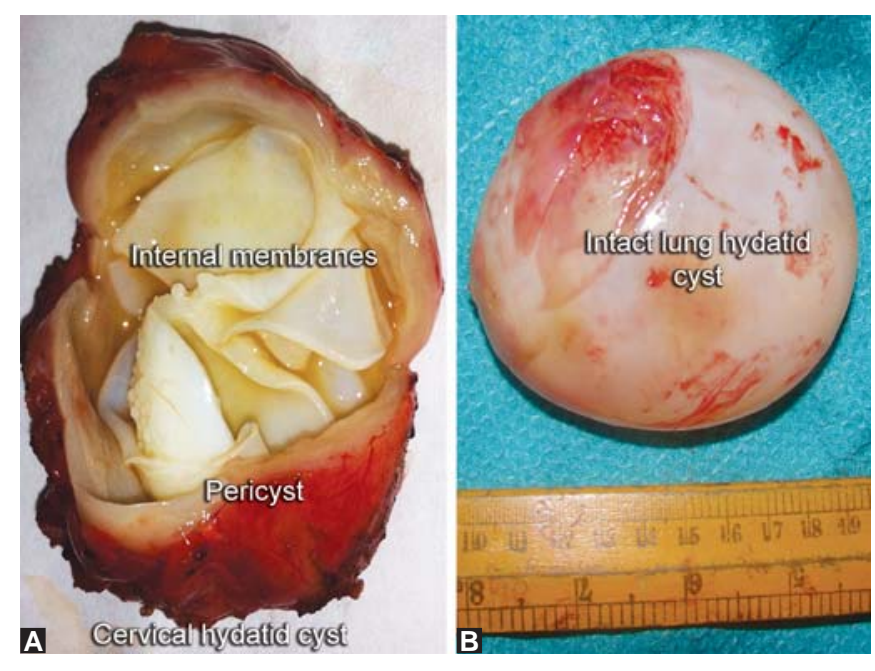

Figs 3A and B: Excised cervical and lung hydatid cysts

An extrahepatic lesion always warrants a thorough search for lesions in other sites.

Though hydatidosis of the neck is rare, we assert that it should be considered as one of the possible diagnosis for any cystic lesion in the neck especially in areas endemic to hydatidosis.

Hydatidosis of the thyroid warrants a thyroidectomy. In our case, the cyst was at the inferior pole of the gland only and not within the lobes and hence a thyroidectomy was avoided.

\section{REFERENCES}

1. Barrett NR, Thomas D. Pulmonary hydatid disease. Br J Surg 1952;40(161):222-44.

2. Bickers WM. Hydatid disease of the female pelvis. Am J Obstet Gynecol 1970;107(3):477-83.

3. Aletras H, Symbas PN. Hydatid diseases of the lung. In: Shields YW, LoCicero J, Ponn RB (Eds). General thoracic surgery (5th ed). Philadelphia: Lippincott Williams and Wilkins 2000;1: 1113-14.

4. Avcu S, Unal O, Kotan C, et al. Submandibular and thyroid gland involvement of hydatid cysts: A very rare association with percutaneous treatment. Diagn Interv Radiol 2010;16(3):251-54.

5. Azendour I, Boulaih M, Ayoubi A, et al. Primary hydatid cyst of the thyroid gland. Int J Otolaryngol 2011;2011:713089.
6. Mitra S, Kundu S, Das S, et al. Mediastinal hydatid disease: An unusual presentation. Indian J Chest Dis Allied Sci 2010;52(4): $245-47$.

7. Ziyade S, Soysal O, Ugurlucan M, et al. Pancoast hydatid cyst leading to Horner's syndrome: Thoracic hydatidosis. Heart Lung Circ 2009;18(5):363-64.

8. Filice C, Pirola F, Brunetti E, et al. A new therapeutic approach for hydatid liver cysts: Aspiration and alcohol injection under sonographic guidance. Gastroenterology 1990;98:1366-68.

\section{ABOUT THE AUTHORS}

\section{Nischal Rajendra Pandya (Corresponding Author)}

Senior PG Registrar, Department of Thoracic Surgery, Christian Medical College and Hospital, Vellore, Tamil Nadu, India, Phone: 08903568935, e-mail: nischalrp@yahoo.co.in

\section{MJ Paul}

Professor and Head, Department of Endocrine Surgery, Christian Medical College and Hospital, Vellore, Tamil Nadu, India

\section{Lalit Choudhry}

Assistant Professor, Department of Thoracic Surgery, Christian Medical College, Vellore, Tamil Nadu, India

\section{Birla Roy Gnanamuthu}

Associate Professor, Department of Thoracic Surgery, Christian Medical College and Hospital, Vellore, Tamil Nadu, India

\section{Kochu Krishnan}

Senior PG Registrar, Department of Thoracic Surgery, Christian Medical College and Hospital, Vellore, Tamil Nadu, India

\section{Joshua Franklyn}

Registrar, Department of General Surgery, Christian Medical College and Hospital, Vellore, Tamil Nadu, India

\section{Jyoti Prasad Kalita}

Assistant Professor, Department of Thoracic Surgery, Christian Medical College and Hospital, Vellore, Tamil Nadu, India 